\title{
Avoidable Errors in Health Policy Analysis
}

\author{
David A. Asch, MD, MBA, John C. Hershey, PhD
}

C ost-effectiveness analysis, decision analysis, and other analytic methods are frequently used to help make health policy decisions or to guide clinical practice. Those who have struggled to promote the application of these techniques to clinical problems are probably gratified that they have taken hold so strongly in only a few decades. But with this wider appeal comes the risk that important basic assumptions about the underlying methods will be overlooked. Ten years ago, for example, Doubilet, Weinstein, and McNeil warned against the overuse of the term "cost-effective" in medicine, and suggested that the term ought to be used less frequently and more precisely. ${ }^{1}$

In this article, we present a related plea that the methods of quantitative health policy analyses, and their presentation, should better reflect their clinical application. Just as the term "cost-effective" can be overused, there is a related tendency for the methods of these analyses to take on a life of their own. Although the products of these analyses are often so persuasive, these analyses are useful only if they represent the underlying clinical problems accurately.

We describe three different kinds of errors that can lead some health policy analyses to misrepresent the clinical reality they are meant to reflect. In describing these errors, we hope not only to improve the way these analyses are performed and presented, but also to help those clinicians and policy makers who interpret and sometimes implement the findings of these analyses make better recommendations and decisions.

\section{PATIENT OR POPULATION PERSPECTIVES}

One area frequently overlooked in performing or interpreting policy analyses is that results are typically re-

Received from the Veterans Affairs Medical Center, Philadelphia, Pa. (DAA); Division of General Internal Medicine (DAA) and Center for Bioethics (DAA, JCH), University of Pennsylvania School of Medicine; Leonard Davis Institute of Health Economics, University of Pennsylvania (DAA, JCH); and Department of Operations and Information Management, The Wharton School, University of Pennsylvania, Philadelphia, Pa. (DAA, JCH).

Address correspondence and reprint requests to Dr. Asch: Leonard Davis Institute of Health Economics, University of Pennsylvania, 3641 Locust Walk, Philadelphia, PA 19104-6218. ported at a population level that disguises variations in outcomes or distributions of outcomes across individuals. We have argued previously that the tendency of some authors to report only population outcomes makes clinical strategies appear less risky in theory than they are in practice. ${ }^{2}$ Indeed, differences between the population perspective embodied in some quantitative analyses and the individual perspective assumed by clinicians caring for patients one by one may explain some clinicians' resistance to these analyses and the guidelines developed from them.

A result presented in terms of a cost per year of life saved, for example, typically represents a population mean and says little about the distribution of possible individual outcomes around that mean. Policy analyses usually assume an aggregate perspective whereby the bad outcomes realized by some patients are offset by the good outcomes realized by others. These analyses do not consider equity. The potential importance of equity concerns is illustrated in the following example.

Imagine two individuals alone on an island who learn that one of two strains of a deadly virus may be approaching. There is a $20 \%$ chance that strain A will arrive, a $20 \%$ chance that strain B will arrive, a $60 \%$ chance that neither strain will arrive, and no chance that both strains will arrive. If either strain arrives, all unimmunized individuals will die from the virus. Vaccines specific for each virus are available, and each confers complete immunity for that specific strain. Unfortunately, island resources are sufficient to produce only two doses of vaccine. What should be done?

Four immunization strategies are possible. ${ }^{3}$ (I) Vaccinate both individuals against the same strain. (II) Vaccinate each individual against a single strain, deciding which strain to use for each person independently. (III) Vaccinate one individual against strain $\mathrm{A}$, and the other against strain B. (IV) Vaccinate one individual against both strains, and the other against none (perhaps by flipping a coin to decide which individual gets the vaccines). These policy choices each use the same resources, but distribute those resources differently. The potential clinical outcomes of these four strategies are presented in Figure 1, from both individual and aggregate perspectives.

In strategy I, the fates of the two individuals are perfectly positively correlated. If they receive the vaccine for strain A, but strain B arrives, both die. There is a $20 \%$ chance this will occur. Otherwise, both live. In contrast, the outcomes are independent in strategy II. As a result, 


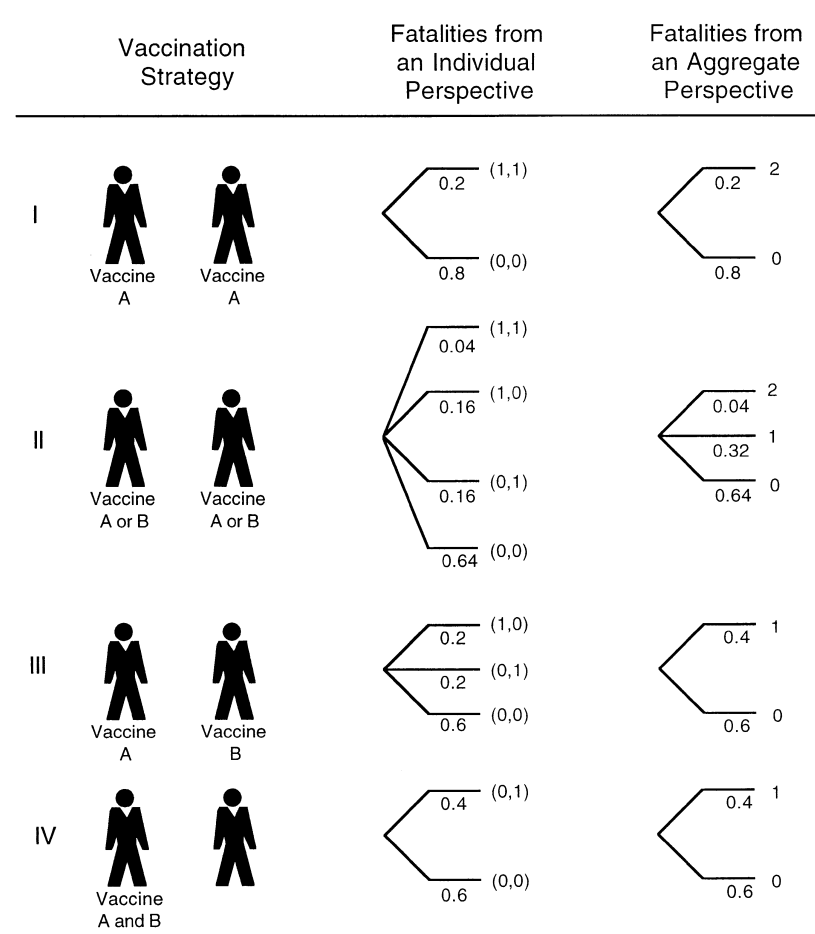

FIGURE 1. Four alternative strategies for allocating two doses of vaccine to two islanders. The center column shows how the risks are distributed to each individual: the notation $(1,1)$ indicates that both individuals die; the notation $(0,1)$ indicates that only the second individual dies, and so on. The right column shows the risks in aggregate, without regard to the distribution of fatalities across individuals. (Adapted from Keeney and Winkler. $)^{3}$

in strategy II, none, either, or both of the islanders might die, with the respective probabilities indicated. In strategy III, the outcomes are as negatively correlated as possible, so that if one dies then the other survives. Under this circumstance, at most one islander will die. In strategy IV, a single individual bears all the risk and, again, at most one islander will die.

Real problems in health policy never approach such simplicity. The differences among these choices do, however, illustrate genuine trade-offs. From an individual perspective, strategies I, II, and III pose identical risks. In each case, each islander faces an individual risk of death of $20 \%$. Islanders who care only about their own survival will be indifferent among these three choices. From an aggregate perspective, however, the strategies provide different levels of risk, because the distributions of total fatalities differ. Strategy III provides the least risk because the outcomes of the two islanders are negatively correlated, so at most one islander can die. Strategy I is the riskiest strategy, and strategy II is somewhere in between. Despite the apparent inequity of strategy IV, from an aggregate perspective it is equivalent to strategy III.

Individuals and policy makers may have different preferences across these alternative strategies, but a tradi- tional cost-effectiveness analysis will not distinguish among them. All consume the same resources (two doses of vaccine), and all result in the expectation of 0.4 deaths. Because of their population perspective, policy makers might prefer strategy III to II, and II to I, in order to reduce aggregate risk. They might also prefer all of these to strategy IV in order to increase equity. They might even choose a program with a higher expected number of fatalities if that is the price they must pay to reduce aggregate risk. In fact, substantial proportions of members of the public, bioethicists, and decision analysts will choose strategies worse for individuals on average if those strategies also spread the opportunities for benefit more evenly. ${ }^{4}$

Because distributions are often important to clinicians and patients, those who perform these analyses, and those who publish, or use them, should insist that results be reported also at the individual level and not only at the population level. To do the former, investigators should describe the distribution of outcomes that individual patients are likely to face, not merely some central tendency reflecting a population trend that perhaps no one will face. In the fanciful example of the islanders, each strategy has the same overall cost-effectiveness. But so much more is learned about the different choices when the distribution of possible outcomes for each is revealed.

\section{PROBLEMS OF REFERENCE POINT}

Another error that can arise when clinical outcomes are evaluated stems from the failure to stick to a fixed reference point when making comparisons. In particular, whenever outcomes are reported in relative terms-for example, "lives saved," "deaths avoided," "dollars saved," or "increased costs"-there is the risk that the point of reference for these relative outcomes will slip, leading to inaccurate results. The error can be exceedingly difficult to detect and can have devastating effects. Moreover, researchers are not the only ones vulnerable to this error. It can arise also as clinicians consider alternative treatments for patients.

Elsewhere, we have referred to this as the "three salesmen" problem, after a classic mathematical conundrum. ${ }^{5}$ But reference point errors arise in many settings. Consider the news vendor problem, a classic inventory problem in the operations research literature. ${ }^{6}$ A news vendor purchases newspapers at a cost of 30 cents each, and sells them for 50 cents. Newspapers unsold at the end of the day have a salvage value of 20 cents, sold back to the publisher. The news vendor's profits, therefore, depend on how well he estimates demand for the papers. If he orders too many papers, he may lose money because the salvage value is lower than his cost. If he orders too few, he misses out on profits. To make things simple, suppose the news vendor knows that demand will be either for 60 papers or for 40 papers, and that there is an equal chance of either demand. How many papers should he order? 
A standard way to approach this problem is to produce a $2 \times 2$ grid reflecting demand of either 60 or 40 papers, and the news vendor's order for either 60 or 40 papers, as shown in Figure 2. The profits under each of the four conditions are calculated, and the probabilities of each demand level are estimated. One can then decide the best choice for the news vendor, at least from a risk-neutral perspective. The news vendor makes 20 cents on each sold paper, so if he orders 60 and the demand is 60 , he makes $\$ 12$. If he orders 60 and the demand is 40 , he makes $\$ 8$ on the 40 papers he sold, and loses $\$ 2$ on the 20 papers he sells back to the publisher, for a net profit of $\$ 6$.

What if he orders only 40 papers? Our own experience is that a surprising number of otherwise thoughtful individuals when presented with this question complete the lower left cell incorrectly. They reason correctly that when the news vendor orders 40 papers and the demand is 40 papers, the profit is $\$ 8$. But they reason incorrectly that when the demand is 60 papers, the profit is $\$ 4: \$ 8$ for selling 40 papers at a profit of 20 cents each, minus the $\$ 4$ the news vendor might have made had he correctly anticipated that he could sell another 20 papers. The correct answer for the lower left cell is $\$ 8$. The $\$ 4$ forgone is reflected in the difference between the $\$ 12$ figure in the upper left and the $\$ 8$ figure in the lower left. People who put \$4 in the lower left count opportunity costs twice.

This error of using $\$ 4$ rather than $\$ 8$ in the lower left cell means that an order of 60 newspapers will appear more attractive than it really is. Similarly, a gambler may mentally double-count the forgone opportunity from passing up a winning bet, and thus may feel that money was lost. Some investors are troubled every time a stock they have bought rises in price; rather than see the gain they

\section{Demand for newspapers}

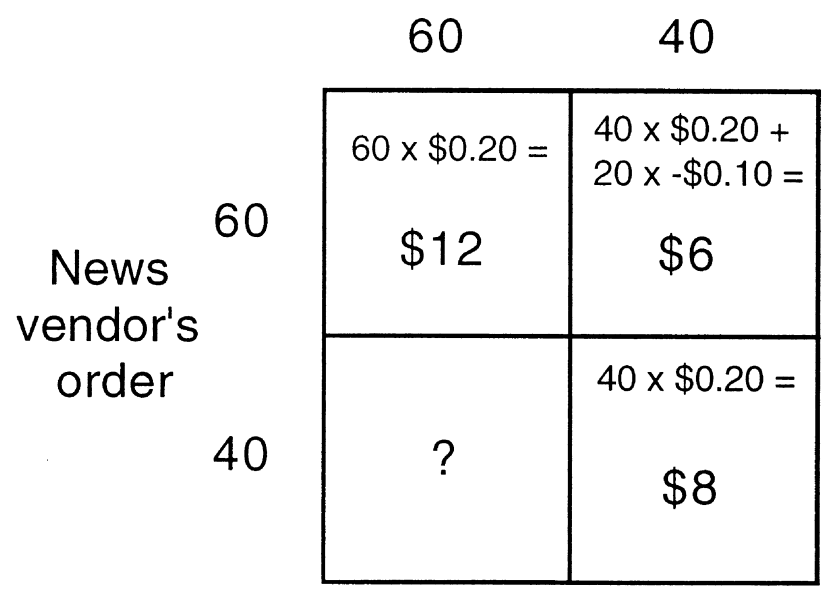

FIGURE 2. The news vendor problem. Newspaper cost is 30 cents, price is 50 cents, and salvage value is 20 cents. Each cell shows the profit for a given order level (supply) and realized demand. What is the news vendor's return in the lower left cell, where the demand for papers exceeds supply? have achieved, they regret not buying more from the start. ${ }^{7}$ Under such circumstances, gamblers and investors may spend more money the next time.

The same error arises in medical contexts. Imagine a clinician is deciding whether to treat a patient with antibiotics for a possible urinary tract infection. To help decide, the same kind of $2 \times 2$ table can be constructed: there is a certain chance the patient has the infection, and a chance the patient does not; and, the clinician can prescribe antibiotics or not. Our experience is that when this kind of problem is posed to physicians, many respond that it is a worse outcome when the clinician treats the patient and the patient does not have a urinary tract infection than when the clinician treats the patient and the patient does have a urinary tract infection. When questioned, their reason is not that there may be some other, more dangerous, medical condition. Rather, they reason that the mistake of prescribing something unnecessary further detracts from the value to the patient. In doing so, they double-count the same way as those responding incorrectly to the news vendor problem. The correct answer is often revealed when one considers that antibiotics are prescribed in both cases, and it should be better that the patient does not have a urinary tract infection than has one. But if this error is not understood, clinicians may be less inclined to prescribe antibiotics than they should be. ${ }^{5}$

There are ways to reduce the chance of this error. Whenever results are presented as "lives saved" or some other relative measure, one should always establish a fixed reference point by asking: "Compared with what?" The reference point can be any specific patient outcome. Even more important, investigators engaging in clinical trials, decision analyses, or epidemiologic studies should be careful to hold to this fixed reference point throughout the intermediate steps of an analysis, and never sum relative differences between alternatives too early in the analysis. For example, when evaluating the life-saving effects of one strategy versus another in a fixed population of patients, calculate the number of deaths (a fixed patient outcome) in both strategies and only then decide which strategy saves more lives or avoids more deaths.

\section{PROBLEMS OF REPEATED OR MULTIPLE TESTS}

Many policy analyses model clinical strategies that involve some sort of diagnostic test used to distinguish between patients who should receive a treatment and patients who should not, or who should receive a different kind of treatment. Sometimes a combination of tests is used, in series or in parallel, as for example in a dipstick and microscopic examination of urine, or an exercise stress test and a coronary angiogram. Problems can arise in these analyses if the correlations that may exist between test results are unknown or minimized.

These problems appear as well in clinical practice. Indeed, the simultaneous use of multiple tests in a policy 
analysis reflects the common practice of using multiple tests with real patients. Sometimes, the same test is used repeatedly. For example, patients suspected of having pulmonary tuberculosis (TB) are often asked to produce three sputum samples for microscopic examination for acid fast bacilli (AFB). The decision rule that most clinicians use is that all three of these tests must be negative for the patient to be "ruled out" or, stated another way, that only one of these tests need be positive to make a presumptive diagnosis of pulmonary TB.

When managing such patients it is important to know how the AFB smear performs as a diagnostic test. Many clinicians are comfortable with general notions of sensitivity (the proportion of patients with pulmonary TB who will have a positive AFB smear) and specificity (the proportion of patients without TB who will have negative AFB smears). But what happens to these test characteristics when the test is used repeatedly-for example, every morning for 3 days?

We recently performed a meta-analysis of 23 articles reporting the test characteristics of the microscopic examination of sputum in suspected pulmonary TB and found that the sensitivity of a single AFB smear is about $65 \%$, and the specificity is about $96 \% .{ }^{8}$ But we could find only a single publication that provided an assessment of the sensitivity of the test used repeatedly, ${ }^{9}$ as it is used in clinical practice, and none that reported the specificity in such a setting. If the sensitivity of one AFB smear is $65 \%$, what is the sensitivity of three AFB smears?

It is easy to calculate the range within which the answer to the above question will fall. If, among patients with $\mathrm{TB}$, the likelihood of having evident AFB in a sputum sample one day is independent of the likelihood on another day, the first test will catch $65 \%$ of those with TB; the second test will catch $65 \%$ of the remainder; the third test will capture $65 \%$ of the remainder after that, and so on. In this case, the overall sensitivity of a three-sample battery would be about $96 \%$. When results are independent from one sample to the next, the sensitivity of an $n$ sample battery when only one of the samples need be positive is given in equation 1 .

$\begin{gathered}\text { Sensitivity of } n \text { sample } \\ \text { independent battery }\end{gathered}=1-(1-\text { Sensitivity of one test })^{n}$

However, if a patient with TB has the same test result each day-in other words, if the results day to day are perfectly positively correlated-no additional information is provided by repeating the test and the joint sensitivity of any $n$-test battery remains 65\%. Not knowing the testto-test correlation means that the range of plausible values for the sensitivity of a three-test battery is between $65 \%$ and $96 \%$, assuming the samples are not negatively correlated. This is a very wide range, given the clinical and public health importance of diagnosing and treating pulmonary TB.

We can ask what the specificity of a three-test AFB battery is, given a $96 \%$ specificity at the level of a single smear. If, among those without TB, the likelihood of a negative sample one day is independent of the likelihood another day, the first test will falsely diagnose $4 \%$ of patients with TB; the second test will falsely diagnose $4 \%$ of the remainder; and the third will falsely diagnose $4 \%$ of the remainder after that, for a joint specificity of approximately $88 \%$. In general, the specificity of an $n$-sample independent battery when only one of the samples need be positive is given in equation 2 .

$$
\begin{aligned}
& \text { Specificity of } n \text { sample } \\
& \text { independent battery }
\end{aligned}=(\text { Specificity of one test })^{n}
$$

In this case, not knowing the correlation among those without the disease creates a plausible range of $88 \%$ to $96 \%$ for the specificity of the three-test battery, again assuming the samples are not negatively correlated.

In summary, one must have a sense of the test-totest correlation to be able to determine the sensitivity and specificity of a multiple-test strategy from the test characteristics of the components. ${ }^{10-12}$ But what may be even less well recognized is that these correlations may not be the same among diseased and nondiseased patients; indeed, it is unlikely that they would be. For example, the correlation between AFB smears among patients with TB may be related mostly to patient characteristics. Some patients have high burdens of mycobacteria, open cavitary pulmonary lesions, or superior abilities to expectorate sputum. Each of these patient characteristics could lead to a higher sensitivity for the diagnostic test, and each of these characteristics would be expected to be preserved from day to day. But reasons for a high correlation among nondiseased patients might have more to do with the clinical laboratory. Perhaps a technician routinely overreads, or the lens or laboratory is contaminated. Politser has observed both low disease/high nondisease group correlations, and high disease/low nondisease group correlations. ${ }^{13}$

Without knowing test-retest correlations separately for both diseased and nondiseased patients, it is impossible to determine how a test will perform when applied more than once, or how two different tests will perform when used in combination. Imagine a study of a group of patients, $50 \%$ of whom have $\mathrm{TB}$, that reports an overall test-retest correlation of 0.6 for the AFB test. It is incorrect to assume that the separate correlations among those with and without TB are also 0.6; indeed, it is impossible for this to be the case. The steeper line in Figure 3 shows the combinations of nonnegative correlations that could produce an overall correlation of 0.6.

What does this mean for a three-test battery? At point A in Figure 3, where the correlation among those with TB is about 0.37 and the correlation among those without TB is 0 , the joint sensitivity for a three-test battery is $85 \%$ and the joint specificity is $88 \%$. At point $\mathrm{B}$, however, sensitivity improves to $90 \%$ and specificity to $96 \%$. The formulas and assumptions for calculating the results from such a three-test battery are described elsewhere. ${ }^{10}$ 


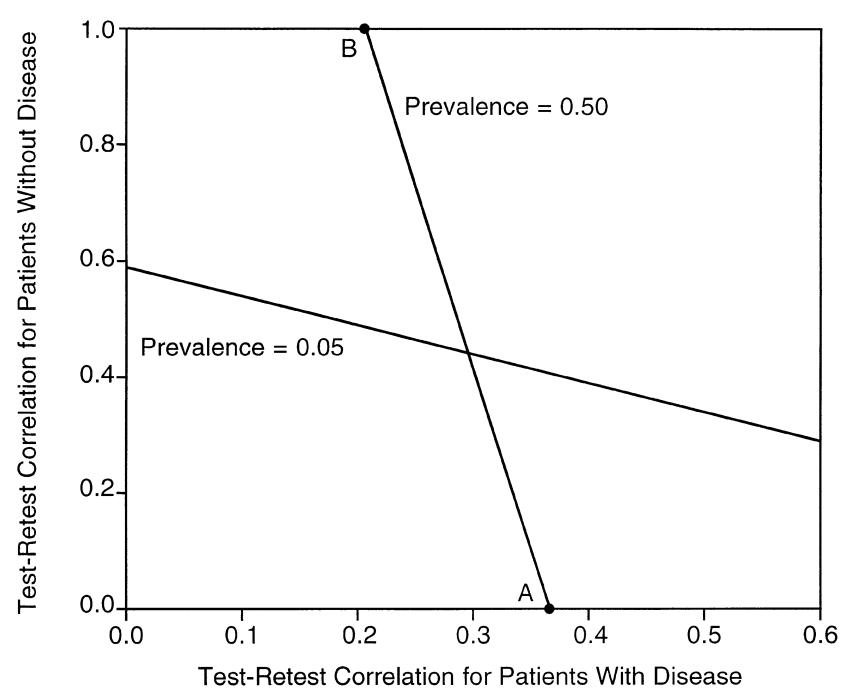

FIGURE 3. Indeterminacy of test-retest correlations. The two lines show the combinations of test-retest correlations that could produce a combined correlation of 0.6 , given disease prevalences of 0.50 and 0.05 . The sensitivity of the test is 0.65 and the specificity is 0.96 . The points $A$ and $B$ are described in the text.

Furthermore, although sensitivity and specificity for a single test are typically independent of disease prevalence, test-retest correlations are not independent of prevalence. Figure 3 also reveals that different combinations of correlations result when the overall correlation is fixed at 0.6 , but disease prevalence falls to $5 \%$. The range of possible correlation values for patients with TB grows, while that for patients without TB shrinks. In other words, test-retest correlations among diseased and nondiseased individuals are essential but rarely reported statistics for interpreting tests used repeatedly or in combination; a single overall correlation says little about the separate correlations for diseased and nondiseased patients; and an unspecified prevalence of disease in the study population makes the translation from an overall correlation to separate correlations even more difficult.

Manipulating probabilities is often challenging, but some standards may decrease the chance that they will be manipulated incorrectly. Those who evaluate diagnostic tests they expect to be used repeatedly or in combination should not be content to report overall sensitivity or specificity. They should, in addition, report separate correlations among diseased and nondiseased groups in their study population, and pay particular attention to how the test is used clinically. At the same time, clinicians and others who use or evaluate diagnostic tests should interpret the results cautiously when these separate correlations are unavailable.

\section{DISCUSSION}

We have described three different kinds of errors that can undermine how policy analyses are conducted, pre- sented, or interpreted. The errors are subtle enough that they can confuse even careful investigators and thoughtful readers. But just as important, these errors reflect disconnections between the methodology of quantitative policy analysis and actual clinical problems faced by individual patients and their physicians.

In the first error, average outcomes reported from an analysis disguise how those outcomes are distributed across individuals in a population. Summary measures reported at the population level are extremely useful to researchers, clinicians, and patients, but in the end they reveal only part of the story. They can also lead to incorrect clinical or policy decisions when issues like fairness or equity across individuals in a population is an independent goal. These errors can be avoided by paying closer attention to the distribution of outcomes that lies behind the average.

In the second error, clinical strategies are evaluated against shifting standards, so that the reported number of "lives lost" or "dollars saved" becomes arbitrary. Often these errors involve some form of double-counting, in which forgone opportunities, or the anticipated regret of choosing the wrong path, count twice in evaluating a decision. These errors are particularly subtle and insidious, but can be avoided with careful attention to a fixed goal, for example, the number of expected deaths.

The third error arises when clinicians or researchers forget that the results of most diagnostic tests they order are not independent of each other. Many people have inaccurate intuitions about probability, and these errors are particularly easy to make. But the medical literature can nevertheless support a higher standard, which is that those reporting the sensitivity and specificity of a diagnostic test should ensure that those reports reflect how that test will be used in actual clinical practice-whether the test is likely to be repeated, for example, or used in combination with other tests.

None of these errors occurs exclusively in policy analysis. Any time results are reported in terms of central tendencies, whether by researcher to clinician, or by clinician to patient, information about distributions is lost. Whenever a clinical approach is described as saving lives, the key question is: Compared with what? Whenever two tests are used in combination, there is the likelihood that they will not behave together as they might behave separately. Quantitative methods offer tremendous promise in framing health policy problems and revealing the likely consequences of alternative approaches. Nevertheless, errors arise when analytic methods become too far removed from clinical practice.

Dr. Asch is the recipient of a Department of Veterans Affairs Health Services Research Career Development Award.

Judy A. Shea, PhD, provided helpful comments about earlier drafts of this manuscript. 


\section{REFERENCES}

1. Doubilet P, Weinstein MC, McNeil BJ. Use and misuse of the term "cost effective" in medicine. N Engl J Med. 1986;314:253-6.

2. Asch DA, Hershey JC. Why some health policies don't make sense at the bedside. Ann Intern Med. 1995; 122:846-50.

3. Keeney RL, Winkler RL. Evaluating decision strategies for equity of public risks. Operations Res. 1985;33:955-70.

4. Ubel PA, DeKay ML, Baron J, Asch DA. Cost effectiveness analysis in the setting of budget constraints: is it equitable? $\mathrm{N}$ Engl $\mathrm{J}$ Med. 1996;334:1174-7.

5. Asch DA, Hershey JC. The three-salesmen conundrum in medicine. Lancet. 1997;350:1393-4.

6. Wagner H. Principles of Operations Research. Englewood Cliffs, NJ: Prentice-Hall; 1969;792-803.

7. Smith A. The Money Game. New York, NY: Random House; 1967, ch 6.
8. Burken MI, Shea JA, Johnson CC, Hershey JC, Asch DA. Comparison of sputum microscopy versus nucleic acid amplification for the presumptive diagnosis of pulmonary tuberculosis: a metaanalysis. Clin Performance Quality Health Care. 1998;6:53-9.

9. Scott B, Schmid M, Nettleman MD. Early identification and isolation of inpatients at high risk for tuberculosis. Arch Intern Med. 1994;154:326-30.

10. Fryback DG. Bayes' theorem and conditional nonindependence of data in medical diagnosis. Comput Biomed Res. 1978;11:423-34.

11. Lachenbruch PA. Multiple reading procedures: the performance of diagnostic tests. Stat Med. 1988;7:549-57.

12. Lui K-J. A note on the effect of the intraclass correlation in the multiple reading procedure with a unanimity rule. Stat Med. 1992; 11:209-18.

13. Politser P. Reliability, decision rules, and the value of repeated tests. Med Decis Making. 1982;2:47-69.

\section{REFLECTIONS}

\section{A Sort of Homecoming}

Leonard had already been diagnosed. HIV. AIDS. Kaposi's sarcoma. Cerebral lymphoma. It was the eighth hospital week now, and John remained at his lover's bedside. Dr. Temple was kind, and every day would bring in a host of wide-eyed medical students and not-so-wide-eyed residents. The doctors and doctors in training would discuss Leonard openly, and John enjoyed feeling a part of the discussion. Leonard used to be a part of the discussion. The discussion used to be exciting. What new medication would they try? Eighty percent show no detectable virus? There is hope! That was 5 weeks ago. With a viral load too high to count and a CD4+ level nearly undetectable, now Leonard lay motionless as the end-stage host overtaken with opportunistic infection. Now when the white coats entered the room the focus was on fluids and electrolytes. "What is his potassium level today? How about calcium? Phosphorus? I's \& O's?"

"The nurse didn't add 'em up, Dr. Temple."

A 'we are doctors and we get things done' look came over Dr. Temple's face as he snatched the doorside chart, quickly took out his pocket calculator, and began frantically punching numbers. It was decided that Leonard would need a G-tube. "We will do surgery tomorrow morning and place a tube into his stomach; that way we can feed him directly and monitor what he gets. It's a common procedure."

"But he can eat!"

"Not enough. His levels are off."

The flock of white coats no longer seemed interested in Leonard. Most of them only half entered the room and John could hear their conversations just outside the door. "So I figure it's sarcoma botryoides."

"Wow, what a great case. All I have is asthma shit."

As the white coats filed out of the room, a lone medical student-the one who always walked right up to the bed, the one who always said hello to both John and Leonard, the one who always made a crack about the weather or the Bulls or the food-stayed behind for but a moment. Leonard asked the student how to disconnect the urine bag and the IV lines.

John lifted Leonard from the soiled sheets and walked down the white tiled hallway and out the door. Leonard was going home.

CURTIS HAGEDORN

Rush Medical College

Chicago, Ill. 